\title{
Femtosecond Laser-Induced Formation of Wurtzite Phase ZnSe Nanoparticles in Air
}

\author{
Hsuan I Wang, ${ }^{1}$ Wei Tsung Tang, ${ }^{1}$ Li Wei Liao, ${ }^{1}$ Pei Shan Tseng, ${ }^{1}$ Chih Wei Luo, ${ }^{1}$ \\ Chu Shou Yang, ${ }^{2}$ and Takayoshi Kobayashi, ${ }^{1,3}$ \\ ${ }^{1}$ Department of Electrophysics, National Chiao Tung University, Hsinchu 30010, Taiwan \\ ${ }^{2}$ Graduate Institute of Electro-optical Engineering, Tatung University, Taipei 104, Taiwan \\ ${ }^{3}$ Advanced Ultrafast Laser Research Center and Department of Engineering Science, Faculty of Informatics and Engineering, \\ The University of Electro-Communications, 1-5-1 Chofugaoka, Chofu, Tokyo 182-8585, Japan
}

Correspondence should be addressed to Chih Wei Luo, cwluo@mail.nctu.edu.tw

Received 27 March 2012; Revised 21 June 2012; Accepted 9 July 2012

Academic Editor: Marinella Striccoli

Copyright (C) 2012 Hsuan I Wang et al. This is an open access article distributed under the Creative Commons Attribution License, which permits unrestricted use, distribution, and reproduction in any medium, provided the original work is properly cited.

\begin{abstract}
We demonstrate an effective method to prepare wurtzite phase ZnSe nanoparticles from zincblende ZnSe single crystal using femtosecond pulse laser ablation. The fabricated $\mathrm{ZnSe}$ nanoparticles are in spherical shape and uncontaminated while synthesized under ambient environment. By controlling the laser fluences, the average size of ZnSe nanoparticles can be varied from $\sim 16 \mathrm{~nm}$ to $\sim 22 \mathrm{~nm}$ in diameter. In Raman spectra, the surface phonon mode becomes dominant in the smaller average particle size with uniform size distribution. The interesting phase transition from the zinc blende structure of ZnSe single crystal to wurtzite structure of ZnSe nanoparticles may have been induced by the ultrahigh ablation pressure at the local area due to the sudden injection of high energy leading to solid-solid transition.
\end{abstract}

The nanometer sized materials have provided an opportunity to study the relation between material properties and size. Due to the uniquely dimension-dependent properties, the nanosized material has the potential for various applications such as nanoelectronics, nanosensors, nanooptronics, and chemical catalyst [1-3]. The nanostructures of zinc selenide $(\mathrm{ZnSe})$, in particular, have attracted considerable attention. ZnSe is an important II-VI semiconductor due to its promising optoelectrical and electrical properties of direct wide band gap $2.68 \mathrm{eV}$ at $300 \mathrm{~K}$. The $\mathrm{ZnSe}$ nanoparticles can be obtained through chemical reduction [4], vapor synthesis [5], or laser ablation method [6]. Generally, crystalline ZnSe exhibits two structural phases, that is, zinc blende and wurtzite [7]. However, the studies on wurtzite phase $\mathrm{ZnSe}$ nanoparticles are few [8-10] owing to that the wurtzite structure is thermodynamically unstable in ambient environments and requires the critical growth conditions. Recently, the femtosecond (fs) laser ablation has been extensively used for material processing [11-15]. Because of the unique features, in fs time-scale pulse duration and high power density $\left(\sim \mathrm{GW} / \mathrm{cm}^{2}\right)$, the high quality nanosized materials can be obtained without thermal effects. For instance, nanospike [12], nanowire [14], and nanoparticle [13, 15] have been produced through this approach. In this study, we present a handy way to fabricate pure ZnSe nanoparticles in metastable wurtzite structure through femtosecond laser ablation technique. The structural phase transition from zinc blende to wurtzite takes place during the processing and the size of nanoparticles is controllable by adjusting the laser fluences. Additionally, the characteristics and formation mechanism of $\mathrm{ZnSe}$ nanoparticles was investigated.

The fs laser pulses used in this study were provided by a Ti: sapphire regenerative amplifier with $800 \mathrm{~nm}$ and $80 \mathrm{fs}$ at a repetition rate of $5 \mathrm{kHz}$. The linearly polarized laser pulses were focused by a planoconvex fused silica cylindrical lens with the focal length of $100 \mathrm{~mm}$ at normal incidence. The spot size on the surface of samples is $2270 \mu \mathrm{m} \times 54 \mu \mathrm{m}$. The pulse energy was varied from 0 to $260 \mathrm{~mJ} / \mathrm{cm}^{2}$ by using 


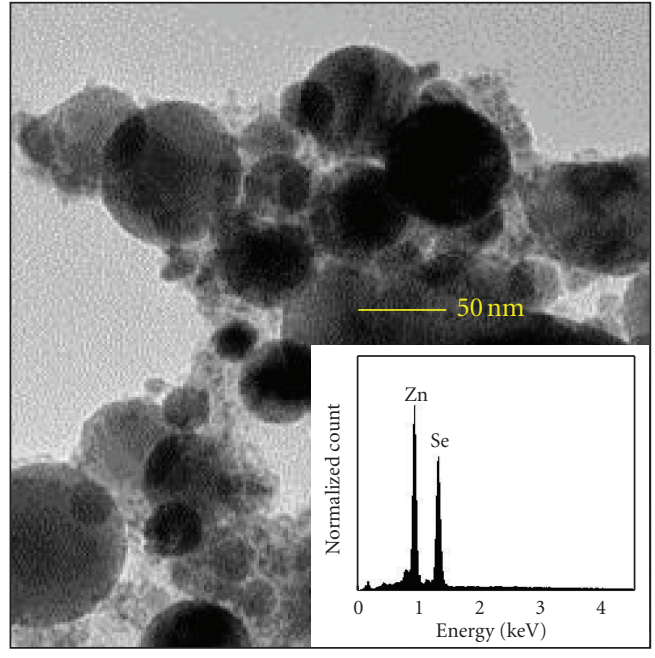

(a)

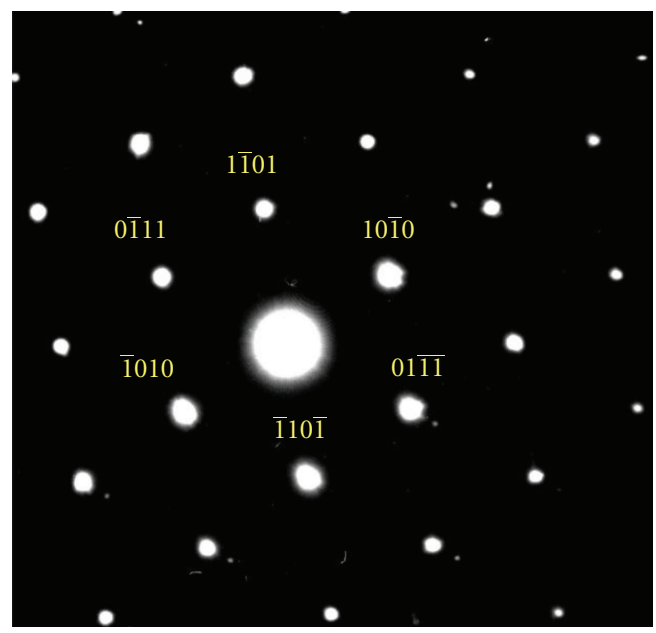

(b)

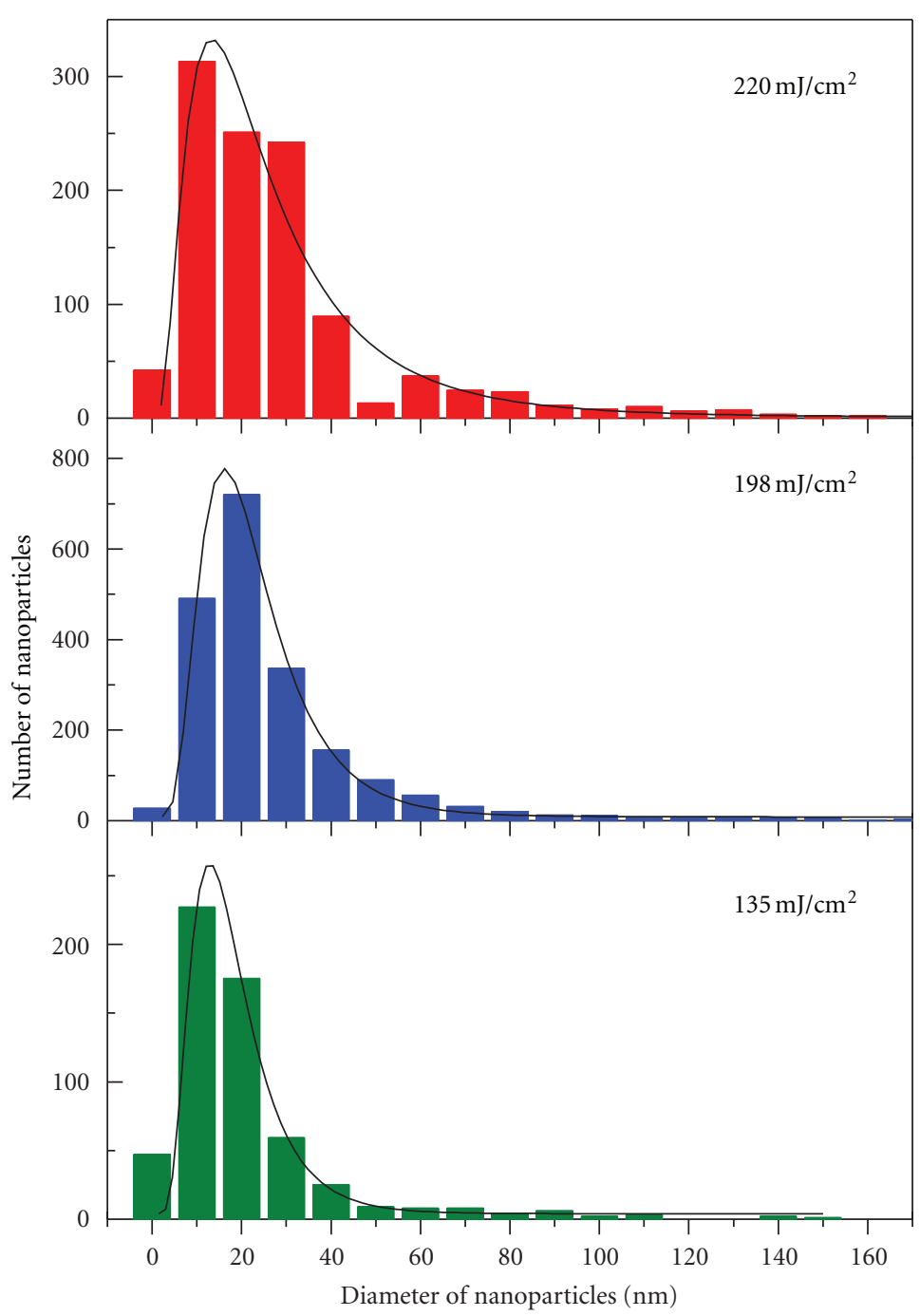

(c)

FIGURE 1: (a) TEM images of ZnSe nanoparticles fabricated by the fluence of $220 \mathrm{~mJ} / \mathrm{cm}^{2}$. Inset: the EDS spectrum shows the composition of ZnSe nanoparticles. (b) TEM diffraction patterns of ZnSe nanoparticles in (a). (c) Size distribution of ZnSe nanoparticles at various laser fluences corresponding to the TEM images in (a) with an area of $3.2 \mu \mathrm{m} \times 2.6 \mu \mathrm{m}$. The solid lines are the log-normal fitting.

metallic neutral density filters with OD0.1-OD2 (Thorlabs ND series). A double-side polished (100) ZnSe wafer $(5 \mathrm{~mm} \times 5 \mathrm{~mm} \times 0.5 \mathrm{~mm})$ was mounted on a motorized $x-y-z$ translation stage with the scanning speed of $100 \mu \mathrm{m} / \mathrm{s}$. After laser irradiation, a pale yellow powder, that is, $\mathrm{ZnSe}$ nanoparticles, was observed on the surface of a processed ZnSe crystal. Depending on the experimental purpose, the nanoparticles were preserved in two different ways, that is, dissolved in ethanol with ultrasonic waves or picked up with Scotch tape. After removing the ZnSe powder, there were many subwavelength ripples shown on the surface of a laser ablated $\mathrm{ZnSe}$ wafer. The direction of ripples was perpendicular to the polarization of laser beam and laser scanning path which is consistent with the scenario of the interference between incident beam and scattering laser light [16]. The morphology of ZnSe single crystals, before and after laser ablation, was examined using a scanning electron microscope (SEM). Moreover, the X-ray diffraction, the Raman scattering spectra, transmission electron microscopy (TEM), and the selected area electron diffraction were applied to identify the structure and characteristics of laser-induced ZnSe nanoparticles.

Figure 1(a) shows a typical TEM image of ZnSe nanoparticles with the smooth spherical shape. The diameters of ZnSe nanoparticles are in the range of tens nanometer. In the selected area electron diffraction (SAED) pattern, a six-fold symmetry was clearly observed in Figure 1(b). Through the analysis of distance and angles between the nearest diffraction points and the center biggest point, the crystal structure of ZnSe nanoparticles was identified as a hexagonal (wurtzite) and the orientation of each diffraction point was marked in Figure 1(b). Furthermore, the energy dispersive 


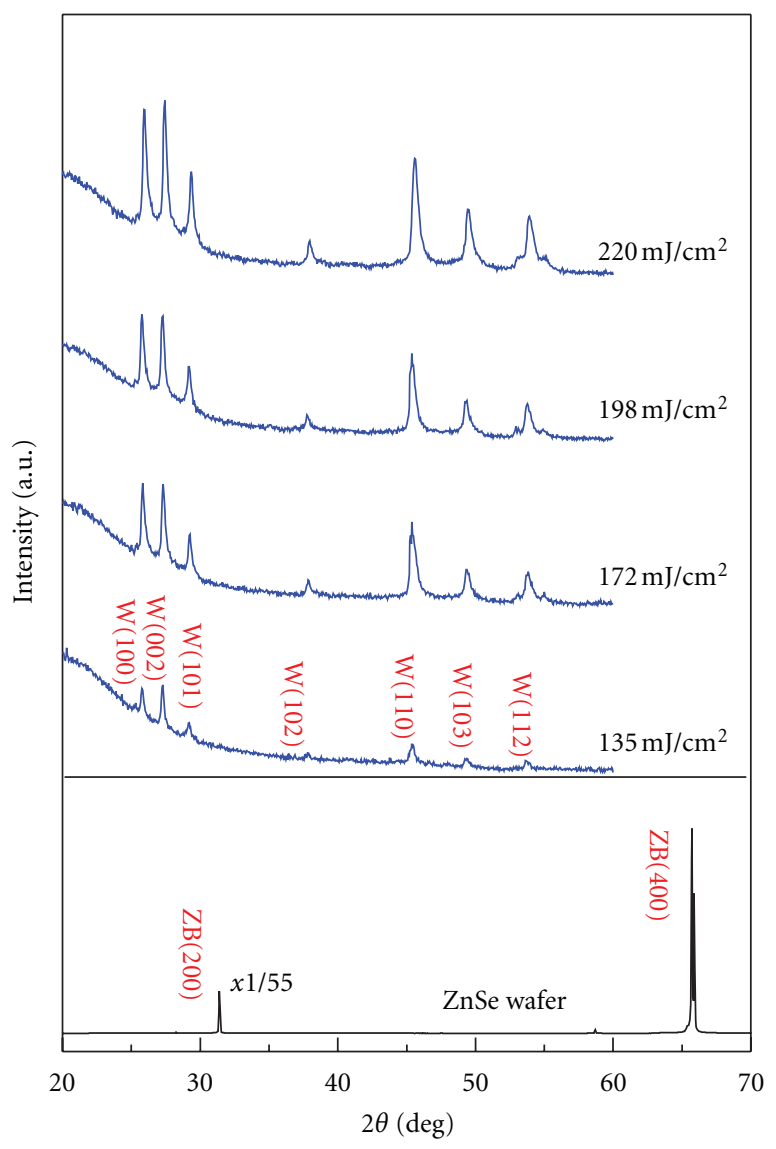

(a)

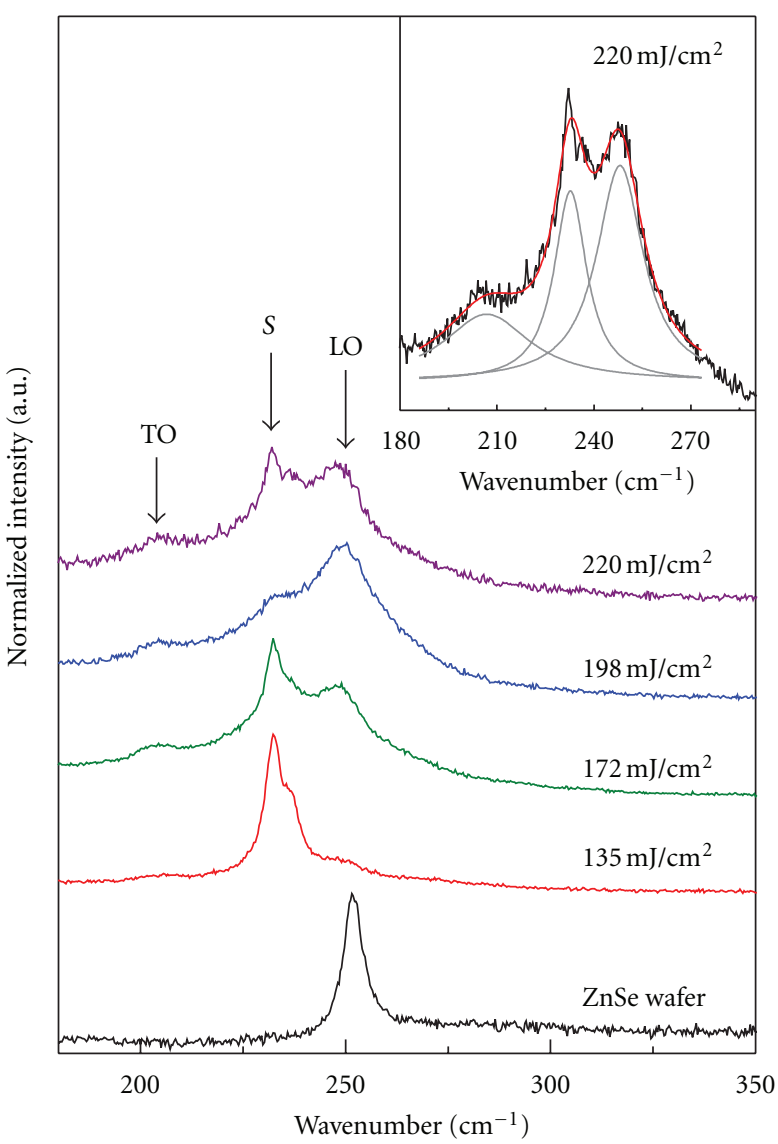

(b)

Figure 2: (a) X-ray diffraction patterns of ZnSe wafer and ZnSe nanoparticles fabricated at various laser fluences. W: Wurtzite. ZB: Zinc blende. (b) Raman spectra of ZnSe wafer and ZnSe nanoparticles fabricated at various fluences. The 633.0 nm laser was used as the excitation light. Inset: the Raman spectra of ZnSe nanoparticles fabricated at fluence of $220 \mathrm{~mJ} / \mathrm{cm}^{2}$ on a large scale with Lorentz peak fitting. TO: transverse optical phonon mode. LO: longitudinal optical phonon mode. $S$ : surface phonon mode.

$\mathrm{X}$-ray spectra (EDX) showed that $\mathrm{Zn}$ and Se are the only elements detected in the laser fabricated nanoparticles and the molar ratio of zinc and selenium is close to $1: 1$ (see the inset of Figure 1(a)). During the femtosecond laser irradiation, the dense plasma is formed on the sample surface via multiphoton absorption [17]. The ablated plume was confined near the laser focused region by surrounding air which prevents other ingredients of air, such as nitrogen, oxygen, and carbon dioxide, to get involved in the formation process of $\mathrm{ZnSe}$ nanoparticles under ambient environment. The size distribution of $\mathrm{ZnSe}$ nanoparticles fabricated at various fluences was analyzed in Figure 1(c). By fitting with the lognormal function, the average diameter of $\mathrm{ZnSe}$ nanoparticles was determined to be approximately $16 \mathrm{~nm}$ in the case of $135 \mathrm{~mJ} / \mathrm{cm}^{2}$. With an increase in the laser fluence to $198 \mathrm{~mJ} /$ $\mathrm{cm}^{2}$ and $220 \mathrm{~mJ} / \mathrm{cm}^{2}$, the average size of the ZnSe nanoparticles slightly increased to $20 \mathrm{~nm}$ and $22 \mathrm{~nm}$, respectively. Although the variation of average size $(\sim 6 \mathrm{~nm})$ is smaller than the statistic bar width of $10 \mathrm{~nm}$ in Figure 1(c) (determined by the variation of average size of $\mathrm{ZnSe}$ nanoparticles under same fluences, which is caused by the fluctuations of laser fluences, pulse durations, spectra, and so on.), the higher fluence indeed generate more ZnSe nanoparticles with larger size. This indicates that the size distribution of $\mathrm{ZnSe}$ nanoparticles can be controlled by adjusting laser fluence and increases as the laser fluence rises. Furthermore, the generation rate of $\mathrm{ZnSe}$ nanoparticles using fs laser pulses is approximately $3.63 \times 10^{10} \mathrm{~s}^{-1}$ (or $7.26 \times 10^{6}$ per pulse) with a fluence of $135 \mathrm{~mJ} / \mathrm{cm}^{2}$. For the higher fluence of $220 \mathrm{~mJ} /$ $\mathrm{cm}^{2}$, the generation rate of $\mathrm{ZnSe}$ nanoparticles increased by one order of magnitude to $3.63 \times 10^{11} \mathrm{~s}^{-1}$ (or $7.26 \times 10^{7}$ per pulse).

In Figure 2(a), X-ray diffraction patterns revealed the structures of a ZnSe single-crystal wafer and the ZnSe nanoparticles fabricated under various laser fluences are zinc blende and wurtzite, respectively, according to the JCPDS card no. 80-0021 $(a=5.618 \AA)$ and no. 80-0008 ( $a=$ $b=3.974 \AA, c=6.506 \AA$ ) for ZnSe. It can be clearly seen that the zinc blende phase of a ZnSe single-crystal wafer has been transferred to the wurtzite phase in the $\mathrm{ZnSe}$ nanoparticles. Because wurtzite $\mathrm{ZnSe}$ is a metastable phase under ambient conditions, it can only be observed under high pressure and high temperature conditions. However, wurtzite $\mathrm{ZnSe}$ nanoparticles can be easily and reliably 
achieved using femtosecond laser ablation as demonstrated in this study. Additionally, the room-temperature Raman scattering spectra of samples was measured by micro-Raman system as shown in Figure 2(b). The typical Raman scattering peaks of ZnSe nanoparticles were clearly observed from 200 to $250 \mathrm{~cm}^{-1}$ which are rather different from that of $\mathrm{ZnSe}$ single crystal wafer. Moreover, there was no vibration mode due to impurities observed in the Raman spectra which is consistent with the above EDX results. A look at the Raman spectra in the case of $220 \mathrm{~mJ} / \mathrm{cm}^{2}$ can be fitted well by three Lorentzian lines located at 250,232, and $203 \mathrm{~cm}^{-1}$. The peaks at 203 and $250 \mathrm{~cm}^{-1}$ are attributed to the transverse optical (TO) phonon mode and longitudinal optical (LO) phonon mode of ZnSe, respectively [18, 19]. Another peak located at $232 \mathrm{~cm}^{-1}$ between the LO and TO phonons is assigned to be the surface phonon mode $(S)$ of nanoparticles. This phenomenon, appearance of surface phonon mode, can be decidedly observed in small-sized material $[9,20]$ due to the high surface to volume ratio and the dominant surface properties. In order to figure out the relation between TO phonon mode and surface phonon mode, the theoretical expression was used as follows [20]:

$$
\frac{\omega_{S}^{2}}{\omega_{T}^{2}}=\frac{\varepsilon_{0}+\varepsilon_{m}((1 / L)-1)}{\varepsilon_{\infty}+\varepsilon_{m}((1 / L)-1)},
$$

where $\omega_{S}$ and $\omega_{T}$ represent the surface phonon and TO phonon frequency, respectively; $\varepsilon_{0}$ and $\varepsilon_{\infty}$ are static and highfrequency dielectric constant, respectively; $\varepsilon_{m}$ is the dielectric constant of surrounding medium and $L$ indicates the depolarization factor which is related to the particle shape. In the case of $\mathrm{ZnSe}, \omega_{T}=203 \mathrm{~cm}^{-1}, \varepsilon_{0}=8.6, \varepsilon_{\infty}=5.7$, and $\varepsilon_{m}$ is equal to 1 for air surrounding condition [18]. Because the laser-fabricated ZnSe nanoparticles are in spherical shape, according to the TEM image shown in Figure 1(a), the depolarization factor $(L)$ is $1 / 3$. Using (1), the calculated surface phonon mode is located at $238 \mathrm{~cm}^{-1}$ which is consistent with the measured value of $232 \mathrm{~cm}^{-1}$. It is worth to note that the LO, TO, and surface phonon modes are strongly fluence dependent. As the laser fluence decreases, the LO and TO peaks gradually shrink; meanwhile, the surface phonon mode increases. In the case of $135 \mathrm{~mJ} / \mathrm{cm}^{2}$, the surface phonon mode almost dominates the Raman spectra, which is due to the smaller average size and more uniform size distribution as shown in Figure 1(c). Additionally, the small-size nanoparticles also result in the red shift of surface phonon mode.

According to the early researches, $\mathrm{ZnSe}$ transforms from a zinc blende structure to the wurtzite structure when the temperature is above the transition temperature $\left(T_{\mathrm{tr}}\right)$ of $1698 \mathrm{~K}$ [7]. When ZnSe crystals are irradiated by the femtosecond laser pulses, the temperature of electrons and lattice in $\mathrm{ZnSe}$ crystals increases according to the two-temperature model [21]. Because of the much smaller heat capacity in electron subsystem, the increase of electron temperature is dramatically larger than that of lattice. The electron temperature $T_{e}$ can be described by [22]

$$
T_{e}=\left\langle\sqrt{T_{i}^{2}+\frac{2(1-R) F}{l_{s} \gamma} e^{-z / l_{s}}}\right\rangle
$$

where $R$ is the reflectivity at $800 \mathrm{~nm}$ and $F$ is the laser fluences and $\gamma$ is the linear coefficient of heat capacity due to the electronic subsystem. The mean value is taken for the depth $z$ going from the crystal surface down to the skin depth $l_{s} \sim 1.87 \mu \mathrm{m}$ (it was estimated from the nonlinear absorption coefficient $\beta[23])$. In this study, taking $T_{i}=295 \mathrm{~K}, R=0$ (which is assumed to be totally absorbed by $\mathrm{ZnSe}$ ), $F=$ $220 \mathrm{~mJ} / \mathrm{cm}^{2}$, and $\gamma=29.4 \mathrm{~mJ} \mathrm{~mol}^{-1} \mathrm{~K}^{-1}$ [24], we obtain $T_{\mathrm{e}} \sim$ $1200 \mathrm{~K}$. However, for the structure transition in materials, the key factor is the lattice temperature rather than the electron temperature. Thus, we further consider the increase in the transient temperature $\Delta T_{\ell}$ in materials can be estimated according to the relationship of $\Delta T_{\ell}=W /(C \times V)$, where $W$ is the pulse energy, $C$ is the heat capacity, and $V$ is the illuminated volume. For $\mathrm{ZnSe}$ at $300 \mathrm{~K}, C$ is $\sim 1.89 \times$ $10^{6} \mathrm{~J} / \mathrm{m}^{3} \mathrm{~K}$ [7], $V$ is $2.29 \times 10^{-13} \mathrm{~m}^{3}$ (absorption depth $1.87 \mu \mathrm{m}$ estimated from the nonlinear absorption coefficient $\beta$ [23]), and $W$ is in the order of $0.243 \mathrm{~mJ}$ (which is assumed to be totally absorbed by $\mathrm{ZnSe}$ ). Thus, the $\Delta T_{\ell}$ is approximately $560 \mathrm{~K}$, which is far below the structural transition temperature of $1698 \mathrm{~K}$. Therefore, a structural transition could not be induced by the increase in temperature. To identify the mechanism underlying the phase transition of $\mathrm{ZnSe}$ from zinc blende to wurtzite, we further analyzed the influence of "ablation pressure" [25], which has been studied from various perspectives over the past few decades [26, 27]. When solids are irradiated by laser pulses, high-density plasma is formed on the surface of the samples [17]. The compressed plasma in laser driven implosions has been characterized as the ablating or exploding pusher according to the surface ablation pressure and bulk pressure due to the preheating through electrons.

In 2003, Batani et al. [25] derived the shock pressure with the laser and target parameters expressed as

$$
P(\text { Mbar })=11.6\left(\frac{I}{10^{14}}\right)^{3 / 4} \lambda^{-1 / 4}\left(\frac{A}{2 Z}\right)^{7 / 16}\left(\frac{Z \times t}{3.5}\right)^{-1 / 8},
$$

where $I$ is the irradiance on target with the unit of $\mathrm{W} / \mathrm{cm}^{2}$; $\lambda$ is the laser wavelength in $\mu \mathrm{m} ; A$ and $Z$ are, respectively, the mass number and the atomic number of the target; $t$ is the time in ns. Figure 3 shows the effective pressure in the irradiated region with the laser peak power density of $0 \sim 3.0 \times 10^{12} \mathrm{~W} / \mathrm{cm}^{2}$. In this study, the maximum pressure induced by the laser reached approximately $1.5 \mathrm{Mbar}$. According to the studies of Greene et al. in II-VI compounds [28], the solid-solid transition point, that is, the zinc blende-wurtzite phase transition, of $\mathrm{ZnSe}$ is approximately $0.55 \mathrm{Mbar}$. In our experiments, the ablation pressure induced by the femtosecond laser pulses on the ZnSe single crystals was in the range of $1.0 \mathrm{Mbar}$ to $1.5 \mathrm{Mbar}$ as shown in the shadow area of Figure 3. This exceeds the solid-solid transition pressure 0.55 Mbar (the dashed line in Figure 3). Therefore, the wurtzite-phase $\mathrm{ZnSe}$ nanoparticles transferred from the zinc blende phase may be caused by high ablation pressure resulting from the femtosecond laser pulses, and the accompanied increase in surface to volume ratio in the nanoparticles. 


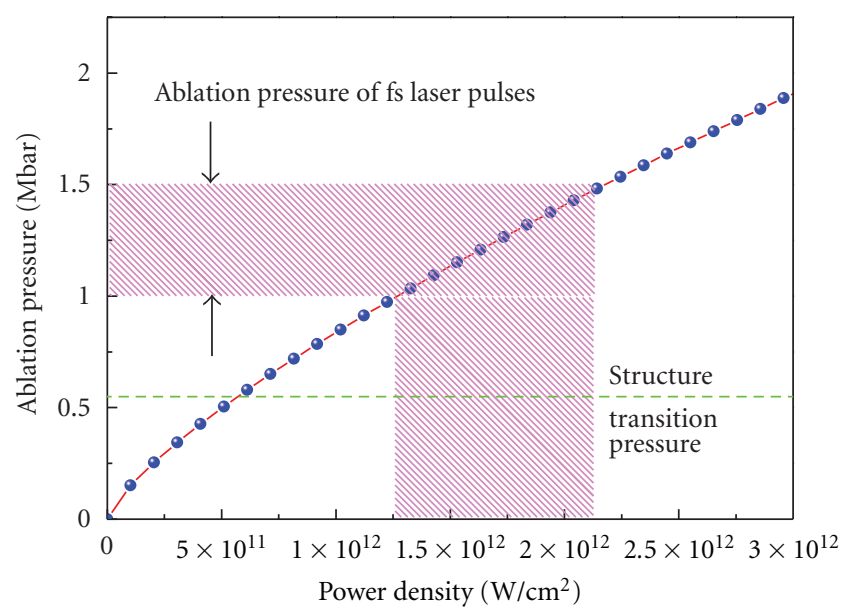

FIGURE 3: Simulated ablation pressure as a function of the laser peak power density according to (3). The shadow area indicates the range of laser peak power density in this study and corresponding ablation pressure. The dashed line represents the pressure of zinc blendewurtzite phase transition, that was obtained from [28].

In summary, we demonstrated a simple and reliable approach to obtain the thermally metastable and pure ZnSe nanoparticles. The spherical-shape wurtzite $\mathrm{ZnSe}$ nanoparticles with the average diameter of $<25 \mathrm{~nm}$ can be well produced through the femtosecond laser ablation technique. While the femtosecond laser pulses are focused on the surface of a $\mathrm{ZnSe}$ wafer in air, the ablated plume cannot expand as rapidly as those in vacuum chamber and then causes an instantaneous high-energy and high-pressure region around the laser focused point; meanwhile, a large amount of $\mathrm{ZnSe}$ nanoparticles was fabricated on the surface of a $\mathrm{ZnSe}$ wafer. During the formation of $\mathrm{ZnSe}$ nanoparticles, the structural phase further changes from the zinc blende phase to the metastable wurtzite phase due to the ultrahigh localized ablation pressure caused by the rapid injection of high laser energy within a femtosecond time scale.

\section{Acknowledgments}

The authors would like to express their sincere appreciation and gratitude to W. T. Chen and Professor Y. J. Hsu for their technical support. This work was supported by the MOEATU program at NCTU and National Science Council of Taiwan, under Grant no. NSC 98-2112-M-009-008-MY3.

\section{References}

[1] M. Bruchez, M. Moronne, P. Gin, S. Weiss, and A. P. Alivisatos, "Semiconductor nanocrystals as fluorescent biological labels," Science, vol. 281, no. 5385, pp. 2013-2016, 1998.

[2] R. S. Friedman, M. C. McAlpine, D. S. Ricketts, D. Ham, and C. M. Lieber, "Nanotechnology: high-speed integrated nanowire circuits," Nature, vol. 434, no. 7037, p. 1085, 2005.

[3] W. C. W. Chan and S. Nie, "Quantum dot bioconjugates for ultrasensitive nonisotopic detection," Science, vol. 281, no. 5385, pp. 2016-2018, 1998.
[4] J. Che, X. Yao, H. Jian, and M. Wang, "Application and preparation of $\mathrm{ZnSe}$ nanometer powder by reduction process," Ceramics International, vol. 30, no. 7, pp. 1935-1938, 2004.

[5] D. Sarigiannis, J. D. Peck, G. Kioseoglou, A. Petrou, and T. J. Mountziaris, "Characterization of vapor-phase-grown ZnSe nanoparticles," Applied Physics Letters, vol. 80, no. 21, pp. 4024-4026, 2002.

[6] K. V. Anikin, N. N. Melnik, A. V. Simakin, G. A. Shafeev, V. V. Voronov, and A. G. Vitukhnovsky, "Formation of ZnSe and CdS quantum dots via laser ablation in liquids," Chemical Physics Letters, vol. 366, no. 3-4, pp. 357-360, 2002.

[7] P. Rudolph, N. Schäfer, and T. Fukuda, "Crystal growth of ZnSe from the melt," Materials Science and Engineering R, vol. 15, no. 3, pp. 85-133, 1995.

[8] Y. P. Leung, W. C. H. Choy, I. Markov, G. K. H. Pang, H. C. Ong, and T. I. Yuk, "Synthesis of wurtzite ZnSe nanorings by thermal evaporation," Applied Physics Letters, vol. 88, no. 18, Article ID 183110, 2006.

[9] C. X. Shan, Z. Liu, X. T. Zhang, C. C. Wong, and S. K. Hark, "Wurtzite ZnSe nanowires: growth, photoluminescence, and single-wire Raman properties," Nanotechnology, vol. 17, no. 22, article 006, pp. 5561-5564, 2006.

[10] L. Jin, W. C. H. Choy, Y. P. Leung, T. I. Yuk, H. C. Ong, and J.-B. Wang, "Synthesis and analysis of abnormal wurtzite ZnSe nanowheels," Journal of Applied Physics, vol. 102, no. 4, Article ID 044302, 2007.

[11] A. P. Joglekar, H. Liu, G. J. Spooner, E. Meyhöfer, G. Mourou, and A. J. Hunt, "A study of the deterministic character of optical damage by femtosecond laser pulses and applications to nanomachining," Applied Physics B, vol. 77, no. 1, pp. 2530, 2003.

[12] M. Y. Shen, C. H. Crouch, J. E. Carey, and E. Mazur, "Femtosecond laser-induced formation of submicrometer spikes on silicon in water," Applied Physics Letters, vol. 85, no. 23, pp. 5694-5696, 2004.

[13] C. W. Luo, C. C. Lee, C. H. Li et al., "Ordered YBCO submicron array structures induced by pulsed femtosecond laser irradiation," Optics Express, vol. 16, no. 25, pp. 20610-20616, 2008.

[14] T. Jia, M. Baba, M. Huang et al., "Femtosecond laser-induced ZnSe nanowires on the surface of a ZnSe wafer in water," Solid State Communications, vol. 141, no. 11, pp. 635-638, 2007.

[15] Y. Nakata, T. Okada, and M. Maeda, "Fabrication of dot matrix, comb, and nanowire structures using laser ablation by interfered femtosecond laser beams," Applied Physics Letters, vol. 81, no. 22, pp. 4239-4241, 2002.

[16] C. Wang, H.-I. Wang, W.-T. Tang, C.-W. Luo, T. Kobayashi, and J. Leu, "Superior local conductivity in self-organized nano-dots on indium-tin-oxide films induced by femtosecond laser pulses," Optics Express, vol. 19, no. 24, pp. 24286-24297, 2011.

[17] A. De Giacomo, M. Dell'Aglio, A. Santagata, and R. Teghil, "Early stage emission spectroscopy study of metallic titanium plasma induced in air by femtosecond- and nanosecond-laser pulses," Spectrochimica Acta B, vol. 60, no. 7-8, pp. 935-947, 2005.

[18] W. Martienssen and H. Warlimont, Springer Handbook of Condensed Matter and Materials Data, Springer, Berlin, Germany, 2005.

[19] Z. D. Hu, X. F. Duan, M. Gao, Q. Chen, and L.-M. Peng, "ZnSe nanobelts and nanowires synthesized by a closed space vapor transport technique," Journal of Physical Chemistry C, vol. 111, no. 7, pp. 2987-2991, 2007. 
[20] S. Hayashi and H. Kanamori, "Raman scattering from the surface phonon mode in GaP microcrystals," Physical Review B, vol. 26, no. 12, pp. 7079-7082, 1982.

[21] S. I. Anisimov, B. L. Kapeliovich, and T. L. Perelman, "Electron emission from metal surfaces exposed to ultrashort laser pulses," Soviet Physics JETP, vol. 39, pp. 375-377, 1974.

[22] C. W. Luo, I. H. Wu, P. C. Cheng et al., "Quasiparticle dynamics and phonon softening in FeSe superconductors," Physical Review Letters, vol. 108, no. 25, Article ID 257006, 2012.

[23] K. Y. Tseng, K. S. Wong, and G. K. L. Wong, "Femtosecond time-resolved Z-scan investigations of optical nonlinearities in ZnSe," Optics Letters, vol. 21, no. 3, pp. 180-182, 1996.

[24] J. A. Birch, "Heat capacities of ZnS, ZnSe and CdTe below 25K," Journal of Physics C, vol. 8, no. 13, Article ID 013, pp. 2043-2047, 1975.

[25] D. Batani, H. Stabile, A. Ravasio et al., "Ablation pressure scaling at short laser wavelength," Physical Review E, vol. 68, no. 6, Article ID 067403, pp. 674031-674034, 2003.

[26] M. H. Key, P. T. Rumsby, R. G. Evans, C. L. S. Lewis, J. M. Ward, and R. L. Cooke, "Study of ablatively imploded spherical shells," Physical Review Letters, vol. 45, no. 22, pp. 18011804, 1980.

[27] J. S. De Groot, K. G. Estabrook, W. L. Kruer, R. P. Drake, K. Mizuno, and S. M. Cameron, "Distributed absorption model for moderate to high laser powers," Physics of Fluids B, vol. 4, no. 3, pp. 701-707, 1992.

[28] R. G. Greene, H. Luo, and A. L. Ruoff, "High pressure X-ray and Raman study of ZnSe," Journal of Physics and Chemistry of Solids, vol. 56, no. 3-4, pp. 521-524, 1995. 

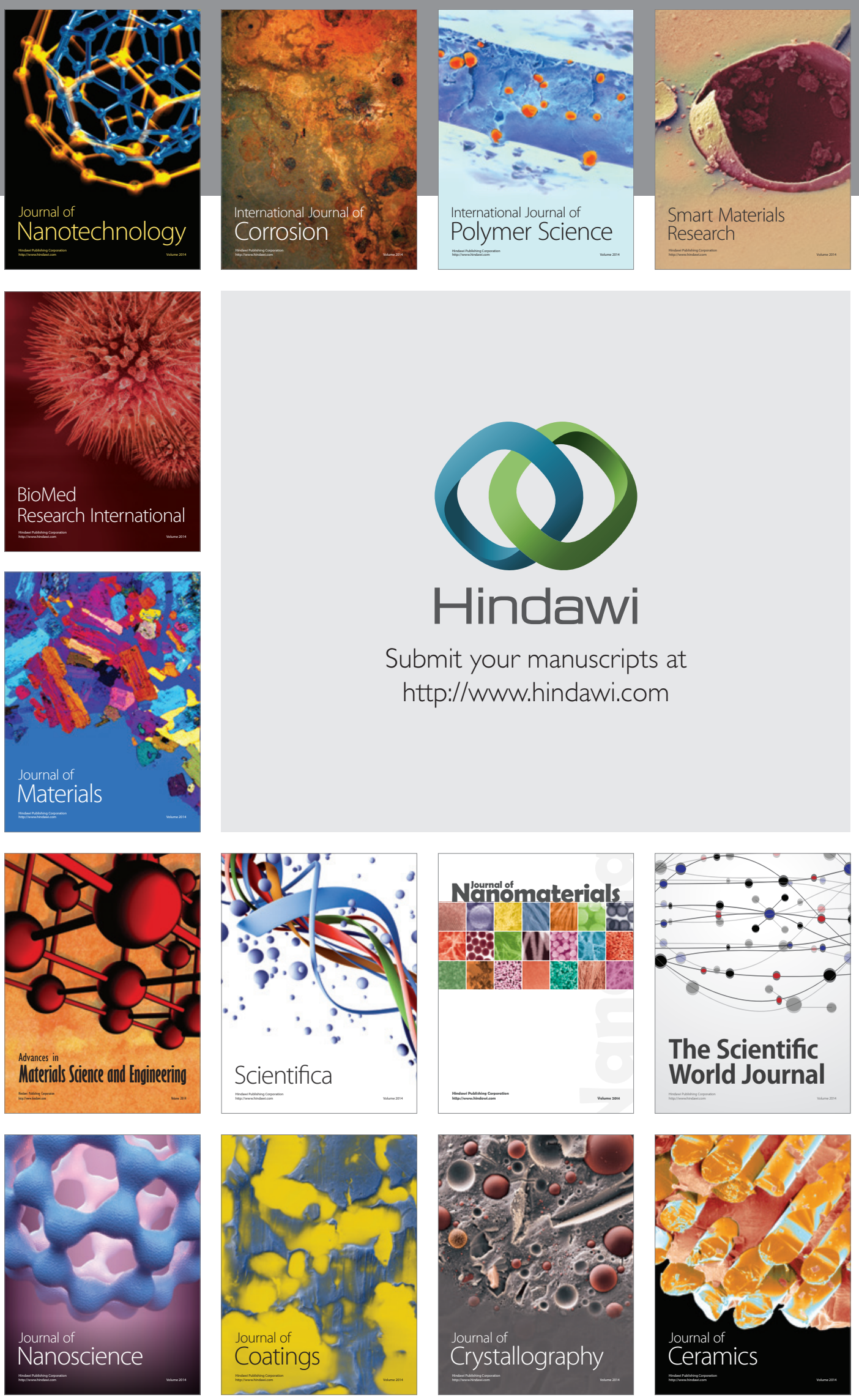

The Scientific World Journal

Submit your manuscripts at

http://www.hindawi.com

\section{World Journal}

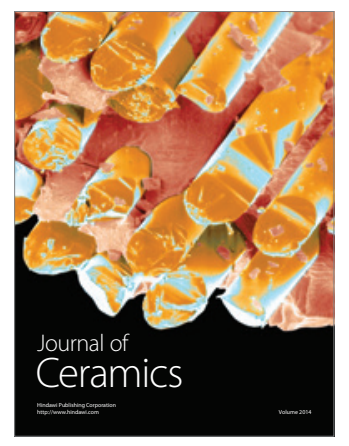

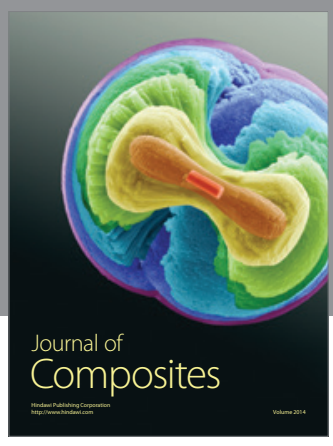
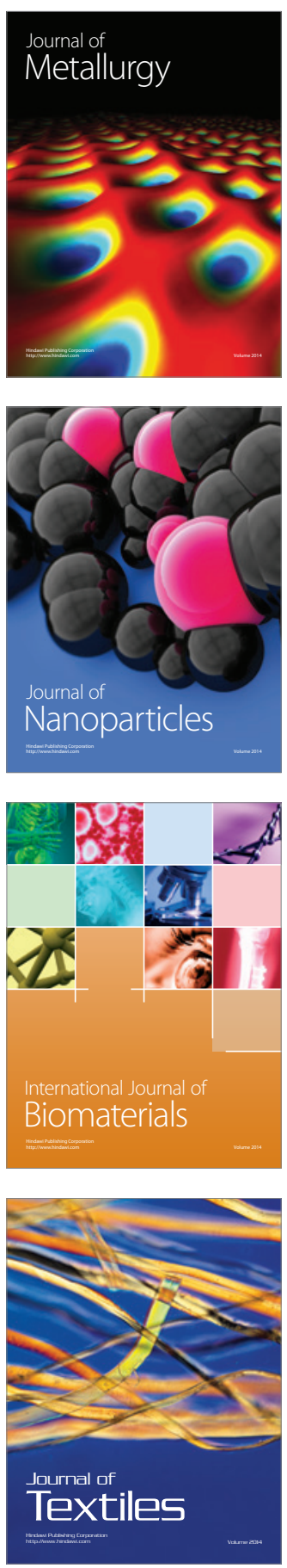